\title{
A phase trial of the oral Lactobacillus casei vaccine polarizes Th2 cell immunity against transmissible gastroenteritis coronavirus infection
}

\author{
Xinpeng Jiang ${ }^{1} \cdot$ Xingyu Hou ${ }^{1} \cdot$ Lijie Tang $^{1} \cdot$ Yanping Jiang $^{1} \cdot$ Guangpeng Ma $^{2}$. \\ Yijing $\mathbf{L i}^{1}$
}

Received: 19 December 2015 /Revised: 20 February 2016 / Accepted: 24 February 2016 /Published online: 28 March 2016

(C) Springer-Verlag Berlin Heidelberg 2016

\begin{abstract}
Transmissible gastroenteritis coronavirus (TGEV) is a member of the genus Coronavirus, family Coronaviridae, order Nidovirales. TGEV is an enteropathogenic coronavirus that causes highly fatal acute diarrhoea in newborn pigs. An oral Lactobacillus casei (L. casei) vaccine against anti-transmissible gastroenteritis virus developed in our laboratory was used to study mucosal immune responses. In this $L$. casei vaccine, repetitive peptides expressed by L. casei (specifically the MDP and tuftsin fusion protein (MT)) were repeated 20 times and the D antigenic site of the TGEV spike (S) protein was repeated 6 times. Immunization with recombinant Lactobacillus is crucial for investigations of the effect of immunization, such as the first immunization time and dose. The first immunization is more important than the last immunization in the series. The recombinant Lactobacillus elicited specific systemic and mucosal immune responses. Recombinant $L$. case $i$ had a strong potentiating effect on the cellular immunity induced by the oral $L$. casei vaccine. However, during TGEV infection, the systemic and local immune responses switched from Th1 to Th2-based immune responses. The systemic humoral immune response was stronger than the cellular immune response after TGEV infection. We found that the recombinant Lactobacillus stimulated
\end{abstract}

Guangpeng Ma

maguangpeng1977@163.com

Yijing Li

yijingli@163.com

1 Department of Preventive Veterinary Medicine, College of Veterinary Medicine, Northeast Agricultural University, Harbin, Heilongjiang 150030, People's Republic of China

2 Agricultural High Technology Department, China Rural Technology Development Center, Beijing 100000, People's Republic of China
IL-17 expression in both the systemic and mucosal immune responses against TGEV infection. Furthermore, the Lactobacillus vaccine stimulated an anti-TGEV infection Th17 pathway. The histopathological examination showed tremendous potential for recombinant Lactobacillus to enable rapid and effective treatment for TGEV with an intestinal tropism in piglets. The TGEV immune protection was primarily dependent on mucosal immunity.

Keywords Transmissible gastroenteritis coronavirus $\cdot$ Phase trial $\cdot$ Lactobacillus casei vaccine $\cdot$ T helper cell

\section{Introduction}

Lactic acid bacteria (LAB) are a group of Gram-positive bacteria that includes species of Lactobacillus, Leuconostoc, Pediococcus and Streptococcus. Consumed for centuries, LAB has enjoyed a long and safe association with humans and animals for healthy food. Over the past decade, there has been increasing interest in the use of LAB as mucosal delivery vehicles. The application of LAB stems from research into effective strategies to deliver vaccine antigens, which may come into contact with the mucosal tissues for the first time, such as the intranasal, oral and genital mucosal surfaces (Lavelle and O'Hagan 2006; Malik et al. 2007). Mucosal delivery of therapeutics or vaccines for chronic diseases and infections of mucosal origin could increase their potency and specificity. Because the mucosal immune system builds an effective IgA barrier in no more than 3 days, contact with the mucosal tissues will neutralize the pathogenic microorganism outside of the host. There are abundant studies in the field of LAB vaccines. One major advantage of the use of $\mathrm{LAB}$ as a delivery vehicle for vaccines is that the bacteria can elicit both antigen-specific secretory immunoglobulin 
(Ig) A and an effective systemic immune response. The specific IgAs have the same function as the neutralizing IgGs. Some candidate $\mathrm{LAB}$ vaccines elicited antigen-specific IgA responses in faeces, saliva or bronchoalveolar and intestinal lavage fluids (Wells and Mercenier 2008). Additionally, lactobacilli are probiotics that may confer health benefits to the host (Bengmark and Gil 2006; Corthesy et al. 2007; Isolauri et al. 2002; Saarela et al. 2000), and there is accumulating evidence that lactobacilli are effective at preventing intestinal disease in both humans and animals due to their ability to inhibit pathogen adhesion to the intestinal wall and prevent inflammatory processes (Blum and Schiffrin 2003; de Vrese and Marteau 2007; Ouwehand 2007; Sartor 2005; Sheil et al. 2007). Because the porcine digestive tract is similar to the digestive tract of human infants with respect to anatomical and histological features and digestive physiology (Kararli 1995; Oswald et al. 2003; Tadros et al. 2003), the pig has been used as an animal model to study gastrointestinal diseases of human infants (Gunzer et al. 2002) and vaccination studies against these diseases.

Coronaviruses (CoVs) comprise a large family of enveloped, positive-stranded RNA viruses that infect a broad range of animal hosts as well as humans. Well-known representatives are porcine transmissible gastroenteritis virus, porcine respiratory $\mathrm{CoV}$, porcine epidemic diarrhoea virus, canine $\mathrm{CoV}$, feline $\mathrm{CoV}$, bovine $\mathrm{CoV}$, human $\mathrm{CoVs}$, severe acute respiratory syndrome-associated $\mathrm{CoV}$, murine hepatitis virus (MHV), avian CoV infectious bronchitis virus (IBV) and turkey $\mathrm{CoV}(\mathrm{TCoV})$. The most famous and critical coronavirus is the Middle East respiratory syndrome virus found in Africa and Asia (Siddell et al. 1983). This study investigates whether mucosal immunization is effective in stimulating a protective immune response against $\mathrm{CoV}$ infection. Transmissible gastroenteritis coronavirus (TGEV), which is a member of the genus Coronavirus, family Coronaviridae, and order Nidovirales, is an enteropathogenic coronavirus that causes highly fatal acute diarrhoea in newborn pigs (Cavanagh 1997). The viral RNA consists of a single strand comprised of three major structural proteins: a phosphorylated nucleoprotein (N protein) and two glycoproteins (the membrane (M) and the spike (S) proteins) (Schwegmann-Wessels and Herrler 2006). The $S$ protein has four sites (A, B, C and D). Both the $A$ and $\mathrm{D}$ sites were demonstrated to induce TGEV-neutralizing antibodies (Di-Qiu et al. 2011); however, the A site is highly glycosylated and thus is not suitable for expression in the $\mathrm{LAB}$ prokaryotic expression vector. Additionally, the different TGEV sites induce different immune responses. Following infection with virulent transmissible gastroenteritis coronavirus, isolated mesenteric lymph node CD4+ T cells mounted a specific proliferative response against infectious or inactivated purified virus upon secondary in vitro stimulation (Anton et al. 1996). The peptide N321 defines a functional T helper epitope that elicits $\mathrm{T}$ cells capable of collaborating with B cells specific for different TGEV proteins (Anton et al. 1995). The most important finding is that oral immunization with a recombinant Lactobacillus vaccine and infection with TGEV elicit various immune responses, such as humoral immunity and cellular immunity. Here, an oral Lactobacillus casei vaccine against anti-transmissible gastroenteritis virus developed in our laboratory was used to study the mucosal immune response (Jiang et al. 2014). In this $L$. casei vaccine, repetitive peptides expressed by $L$. casei (specifically the MDP and tuftsin fusion protein (MT)) are repeated 20 times and the D antigenic site of the TGEV spike (S) protein is repeated six times.

The pig model was developed to study intestinal mucosal immune responses (Ruan and Zhang 2013). Probiotic feed supplementation may benefit the animal host directly by preventing infection and combating the causative agent of the intestinal disorder by balancing the disrupted equilibrium of the enteric flora and augmenting the host's immune responses. However, LAB vaccine has not received national law or certification for human or animal use against coronaviruses. The first clinical trial to use recombinant LAB demonstrated that the containment strategy for L. lactis expressing recombinant IL-10 was effective against Crohn's disease (Braat et al. 2006). Our laboratory has researched the $\mathrm{LAB}$ vaccine for more than one decade, and we have developed many LAB vaccines in the field of piglet diarrhoea (DiQiu et al. 2011; Liu et al. 2011; Qiao et al. 2009; Yigang and Yijing 2008). This study is the last step to obtain new drug certification in China. This Lactobacillus vaccine has been demonstrated to increase the Treg population in the mouse model (Jiang et al. 2014). Tregs effectively depressed $T$ and $\mathrm{B}$ cell proliferation, and some studies demonstrated that this regulation also depressed proliferation in inflammatory bowel disease. Our study investigated whether the recombinant Lactobacillus vaccine gradually increased the Breg and Treg populations during the immunization process at the first step of immunization (unpublished data). The intestinal immune system of the pig maintains its ability to mount an active immune response against pathogens and exhibits tolerance to at least food antigens and probably commensal flora through an extremely complex network of cellular and humoral immune interactions. Consequently, it is important to elucidate the immunological inductive sites of the protective mucosal immune response following oral immunization in pigs.

This vaccine could induce TGEV antibody immune responses in both the humoral and mucosal immune systems. MDP and tuftsin possess substantial immunopotentiating properties and can induce cellular-mediated immune responses upon oral administration in mice. However, their use in oral vaccines against TGEV challenge in the pig host may have different results. Furthermore, the only host (and target of the vaccine) of the transmissible gastroenteritis coronavirus is the weaning piglet. There have been no clear reports 
concerning whether TGEV infection stimulates Th1 or Th2type immune response. The relationship between humoral and cellular immune responses is not clear; moreover, whether the systemic or mucosal lymphoid response will be the primary immune response following oral immunization is unknown. At present, we are not certain which pathway the Lactobacillus vaccine will provoke against TGEV infection. Our study is the first to analyse immunity in response to oral immunization and TGEV infection.

\section{Materials and methods}

\section{Virus, bacterium and cell line}

The L. casei ATCC 39392 strain used in this study was deposited in ATCC and is a plasmid-free strain grown in ManRogosa-Sharpe (MRS) medium (Sigma) at $37{ }^{\circ} \mathrm{C}$ without shaking. The recombinant $L$. casei (PG:612-20MT6D) was generated in previous study (Jiang et al. 2014). Chloramphenicol $(\mathrm{Cm})$ and kanamycin (Sigma) were each used at a final concentration of $10 \mu \mathrm{g} / \mathrm{mL}$. TGEV was previously isolated and purified in our laboratory. Swine testicle (ST) cells were cultured in Dulbecco's modified Eagle's medium (DMEM, Gibco) supplemented with $10 \%$ foetal bovine serum (FBS, Gibco) at $37^{\circ} \mathrm{C}$ with $5 \% \mathrm{CO} 2$. The virus stocks were clarified by centrifugation at $800 \times g$ for $10 \mathrm{~min}$ to remove cell debris, titrated using the cytopathic effect assay and then stored in aliquots at $-80{ }^{\circ} \mathrm{C}$ until needed.

\section{Experimental design}

TGEV-seronegative crossbreed (large white) piglets were obtained from a local breeding farm after birth. The piglets were housed separately in specialized cages that were maintained in sterile stainless steel isolators (five piglets/isolator) and fed commercial sterile milk and water. Four groups $(n=5$ each) of piglets were orally dosed with $10^{10}$ CFU of PG:61220MT6D in $1 \mathrm{~mL}$ of PBS or PBS alone (Jiang et al. 2014); this formulation was used to immunize piglets via an intragastric route in a different immunization protocol. The first group was immunized with PG:612-20MT6D in $1 \mathrm{~mL}$ for priming. The second group was immunized with PG:61220MT6D in $2 \mathrm{~mL}$ for priming. The third group was immunized with PG:612-20MT6D in $2 \mathrm{~mL}$ for 48 consecutive hours. The forth group was immunized with PG:61220MT6D in $1 \mathrm{~mL}$ for 48 consecutive hours. The control group was immunized with PBS. The piglets were handled and maintained under strict ethical conditions according to international recommendations for animal welfare.

Seven days after immunization, serum samples were prepared from collected blood samples. The intestinal mucus was collected by rectal swab and subsequently homogenized for
$30 \mathrm{~min}$ in $400 \mu \mathrm{L}$ of sterile PBS (pH 7.4) containing $0.01 \mathrm{~mol} /$ 1 EDTA-Na2 and then incubated for $12 \mathrm{~h}$ at $4{ }^{\circ} \mathrm{C}$. Clear extracts of all samples were collected by centrifugation at $3000 \times g$ for $10 \mathrm{~min}$ and stored at $-80^{\circ} \mathrm{C}$ with protease inhibitors for subsequent analysis.

\section{Enzyme-linked immunosorbent assay}

Enzyme-linked immunosorbent assay (ELISA) plates were coated for 18 and $12 \mathrm{~h}$ at $4{ }^{\circ} \mathrm{C}$ with full TGEV and the VP4 protein, respectively, which were previously isolated and purified in our laboratory. Cultured ST cells were used as a negative antigen control. After the wells were blocked for $12 \mathrm{~h}$ at $4{ }^{\circ} \mathrm{C}$ with PBS containing $5 \%$ skim milk, the plates were washed three times with PBS + Tween 20 (0.1\%) (PBST). Mucus and serum (diluted 1:50) samples were added to the wells in triplicate and incubated for $1 \mathrm{~h}$ at $37{ }^{\circ} \mathrm{C}$. Afterwards, the plates were washed three times with PBST, and a horseradish peroxidase-conjugated goat anti-pig IgG or IgA antibody (Invitrogen) was added to each well (1:5000) and incubated for an additional $1 \mathrm{~h}$ at $37^{\circ} \mathrm{C}$. After another round of washing, colour development was accomplished using ophenylenediamine dihydrochloride as a substrate, and the absorbance was measured at $490 \mathrm{~nm}$.

\section{Th cell analysis and cytokine detection}

Naive purified spleen T cells $\left(5 \times 10^{5}\right.$ cells $\left./ \mathrm{mL}\right)$ were cultured in 24-well tissue culture plates and stimulated with $1.0 \mu \mathrm{g} \mathrm{mL}^{-1}$ of plate-bound anti-CD4(PE) antibody (Abcam) in complete RPMI 1640 medium. Single-cell suspensions were stimulated in culture with ionomycin $\left(2 \mu \mathrm{g} \mathrm{mL}^{-1}\right)$ in the presence of monensin $(2 \mu \mathrm{M})$ for $4-6 \mathrm{~h}$ of culture. The cells were surface labelled and then fixed, permeabilized and intracellularly labelled with IFN- $\gamma$ and IL-4 antibodies as previously described (Moore-Connors et al. 2013; Zhou et al. 2009). For Th1 and Th2 differentiation, the cells were stimulated in the presence of $10 \mathrm{ng} \mathrm{mL}^{-1}$ antiIFN (FITC) and $10 \mathrm{ng} \mathrm{mL}^{-1}$ anti-IL-4 (FITC) (Abcam) antibodies. The cells were labelled with carboxyfluorescein succinimidylester (CFSE) according to a previous protocol (Jiang et al. 2014). The data were acquired by gating on the $\mathrm{CD}^{+}$cell population with a FACSCalibur cytometer. The sequential loss of CFSE fluorescence was used to measure cell division and proliferation.

\section{Protective efficacy}

Groups were housed in the same facility but separated by room and ventilation system. Pigs in each room were confined by pens on a solid floor that was rinsed daily, fed a balanced diet ad libitum based on weight and provided free access to water. TGEV-challenged pigs received a $5 \mathrm{~mL}$ dose of $1 \times 10^{5}$ 
plaque-forming units (PFU)/mL via oral-gastric gavage on 0 days post-inoculation (dpi). Pigs in the control group were administered volume-matched virus-free cell culture media. The control, immunized and no challenge group pigs were randomly selected for necropsy on the fourth day.

\section{Gross lesion and histopathological examinations}

To assess histological changes in the intestinal tissues, both the intestine and other major organs were examined at necropsy. After $48 \mathrm{~h}$ of fixation in $10 \%$ neutral buffered formalin, tissue sections were trimmed, processed, and embedded in paraffin, sectioned, stained with haematoxylin and eosin (H\&E) and then examined for pathological changes by light microscopy using a model microscope (Olympus, Tokyo, Japan).

\section{Real-time RT-PCR analysis}

Real-time RT-PCR (qRT-PCR) was employed to determine the amount of TGEV and cytokine gene products (ISGs) in rectal swab samples and the intestinal tissues using a CFX96TM Real-Time PCR Detection System (Bio-Rad). Total RNA was extracted from faecal samples and splenic and intestinal tissues using viral RNA extraction and total RNA extraction kits (iNtRON) according to the manufacturer's instructions. The extracted RNA was subjected to real-time qRT-PCR using a One-Step SYBR ${ }^{\circledR}$ qRT-PCR reagent kit (Takara, Shiga, Japan). Following reversetranscription of the viral RNA at $45{ }^{\circ} \mathrm{C}$ for $20 \mathrm{~min}$, the resulting cDNAs were used for real-time PCR amplification. A standard curve was generated by plotting threshold values against serially diluted plasmid DNA encoding the fragment of the TGEV spike protein (Lee et al. 2011). All determinations were performed using data from wells evaluated in duplicate to ensure reproducibility. The copy number of the experimental samples was determined by interpolating the threshold cycle values using the standard curve. Real-time quantitative PCR was utilized to quantify the products of interest (TRL-2, $-4,-9$, IL-4, IL-17, IFN- $\gamma$ and TGF- $\beta$ ) relative to the quantity of messenger RNA (mRNA) in the total RNA isolated from the splenic and intestinal tissues (Dirisala et al. 2013; Kiros et al. 2011); the specific primers are listed in Table 1. The Livak method ( $\Delta \Delta \mathrm{CT}$ method) was used to calculate the fold change compared to the $\beta$-actin gene control. Gene expression data were expressed relative to unimmunized and uninfected piglets.

\section{Th cell analysis after infection}

To phenotype immune cells in the spleen and mesenteric lymph nodes, single-cell suspensions were isolated and labelled with fluorochrome-conjugated antibodies. To determine the cell type and the frequency of IFN- $\gamma$ and IL4-producing Th1 and Th2 cells, single-cell suspensions were stimulated in culture with ionomycin $\left(2 \mu \mathrm{g} \mathrm{mL}^{-1}\right)$ in the presence of monensin $(2 \mu \mathrm{M})$ for $4-6 \mathrm{~h}$. The cells were surface labelled to detect CD4 and then fixed, permeabilized and intracellularly labelled with IFN- $\gamma$ and IL-4 antibodies as previously described (Moore-Connors et al. 2013; Zhou et al. 2009).

\section{Statistical analysis}

Comparison of the piglets' IgA and IgG titres was conducted by a paired $t$ test. The Th cell, cytokine expression and faecal PEDV RNA shedding titres among litters were compared using one way analysis of variance (ANOVA) followed by Duncan's multiple range test.

\section{Results}

\section{Production of mucosal and humoral immunity}

The mucosal immune response of the piglets was evaluated by measuring the IgA response in diluted intestinal lavage fluid post-intragastric immunization. As shown in Fig. 1a, the newborn piglets that received $2 \mathrm{~mL}$ of recombinant Lactobacillus PG:612-20MT6D had the highest mucosal IgA levels after immunization. The IgA levels at $48 \mathrm{~h}$ in the piglets that received $4 \mathrm{~mL}$ were slightly lower than the newborn piglets that received $2 \mathrm{~mL}$ throughout the process. The vaccine doses also provoked systemic immunity based on the serum analysis and elicited specific IgG responses from the immunized piglets (Fig. 1b). Newborn piglets that received $2 \mathrm{~mL}$ of the vaccine also had the highest specific IgG level. The specific IgG titre reached as high as 1:1600. Finally, the antibody kinetics in the serum and intestinal lavage samples from the animals showed that the specific antibody IgG and IgA levels were increased on the seventh and eighth days and the titre was decreased during the last week without immunization.

\section{Activation of T helper cells by recombinant Lactobacillus}

To analyse the effect of recombinant Lactobacillus PG: 61220MT6D on T helper cells, we evaluated T helper cell polarization. Throughout the process, we utilized the model of immunization described here. As shown in Fig. 2, the immune response balance mediated by Th1 and Th2 was broken in favour of Th1-mediated immunity.

\section{Protective efficacy}

The PG:612-20MT6D $/ L$. casei group exhibited $80 \%$ protection within 4 days of challenge with TGEV (PG:612- 
Table 1 Primer sequences used in this study

\begin{tabular}{llll}
\hline Gene & $\begin{array}{l}\text { Sequence }\left(5^{\prime}-3^{\prime}\right) \\
\text { Forward }\end{array}$ & $\begin{array}{l}\text { Sequence }\left(5^{\prime}-3^{\prime}\right) \\
\text { Reverse }\end{array}$ & Accession no. ${ }^{\text {a }}$ \\
\hline TLR2 & ACATGAAGATGATGTGGGCC & TAGGAGTCCTGCTCACTGTA & AB072190 \\
TLR4 & CTCTGCCTTCACTACAGAGA & CTGAGTCGTCTCCAGAAGAT & AB078418 \\
TLR9 & GTGGAACTGTTTTGGCATC & CACAGCACTCTGAGCTTTGT & AB071394 \\
IFN- $\gamma$ & ACTTATTTCTTAGCTTTTCAGCTTTGC & GGCGCCTGGCAGTAAGAG & S63967 \\
IL-4 & CGTGACGGACGTCTTTGCT & CCCGGCAGAAGGTTCCT & X68330 \\
IL-17 & CTCTCGTGAAGGCGGGAATC & GTAATCTGAGGGCCGTCTGG & YB02693 \\
TGF- $\beta$ & CACGTGGAGCTATACCAGAA & TCCGGTGACATCAAAGGACA & U07786 \\
$\beta$-actin & CATCACCATCGGCAACGA & GCGTAGAGGTCCTTCCTGATGT & NC002306.2 \\
TGEV spiked ${ }^{\text {a }}$ & GCAACCATTGGAATCTCATTGAAACCTTCC & ACGTTTAACCGTTGTCTGTGATTCC \\
\hline
\end{tabular}

${ }^{\mathrm{a}}$ The sequences of the two primers were checked using the NCBI Blast Software, and no significant alignment with any other animal virus gene was found

20MT6D/L. casei) (Fig. 3). In contrast, the control group piglets immunized with PBS all died after TGEV challenge. All piglets that died/were killed were emaciated and had yellow faeces coating the skin and hair. In some piglets, the intestinal lumens were filled with large amounts (approximately 50-70 mL) of yellowish foamy fluid. In other piglets, the walls of the small intestine were transparent and thin and the intestinal lumens were empty. No significant gross lesions were observed in other major organs (lung, kidney, liver and heart).

\section{Gross lesions and histopathological examination}

Formalin-fixed intestinal tissue sections from piglets treated with different treatment modalities were blindly analysed for histopathological changes associated with TGEV infection.

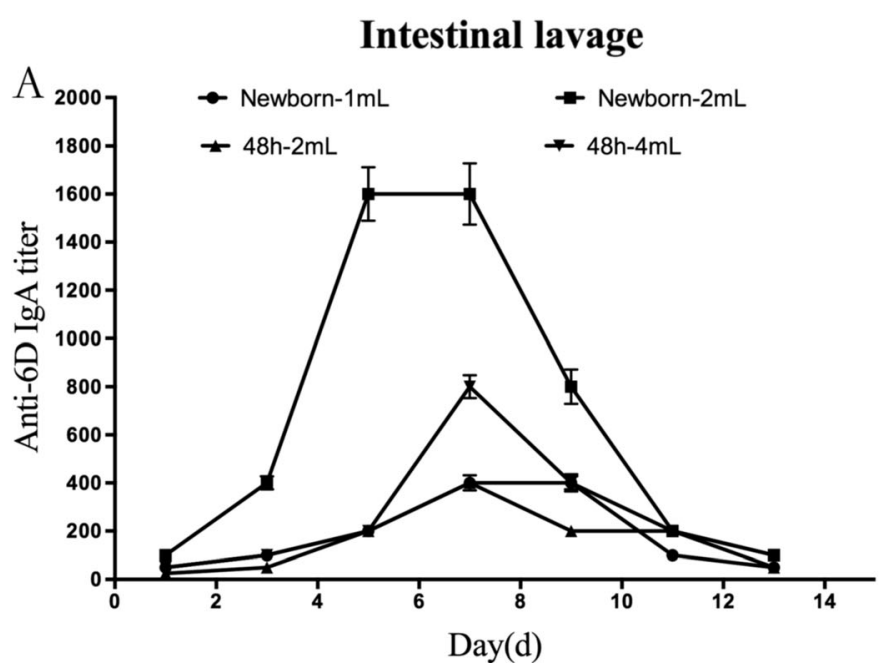

Fig. 1 Specific secretory immunoglobulin (Ig) levels. a Intestinal lavage fluid was collected from the piglets groups administered PG:61220MT6D and PBS on the 13 day of immunization; lines represent the IgA titres \pm standard errors of the means in each group. b Serum was
According to the histopathological analysis, the small intestine samples from the three groups (positive, negative and immunized groups) showed obvious differences. As indicated in Fig. 4, different degrees of pathological changes were detected after infection, especially in the positive group where serious damage was observed. The representative pathological changes included intestinal villi hyperaemia, atrophy and destruction. In the PBS infection group, the jejunum villi were severe atrophied and destroyed, and the ilea exhibited severe lymphocyte proliferation in the lamina propria. The recombinant Lactobacillus group showed jejuna with intact villi but low-grade hyperaemia and lymphocyte proliferation, and the ilea exhibited lymphocyte proliferation in the lamina propria. Both the PBS and vaccine groups had severe inflammatory responses. The negative control piglets that were not infected showed normal histology.

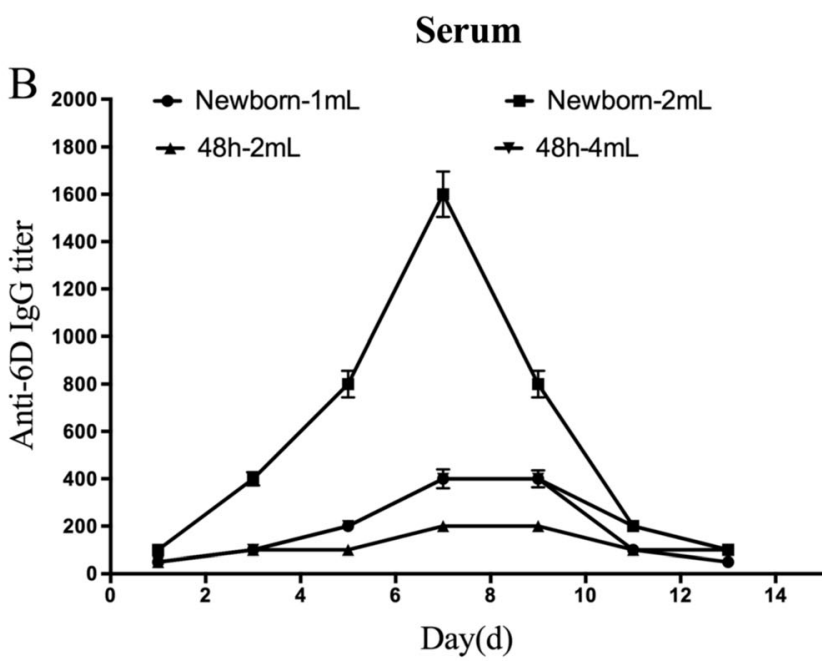

collected from the piglets groups administered PG:612-20MT6D and PBS on the 13 day of immunization; lines represent the IgG titres \pm standard errors of the means in each group 
PG:612-20MT6D

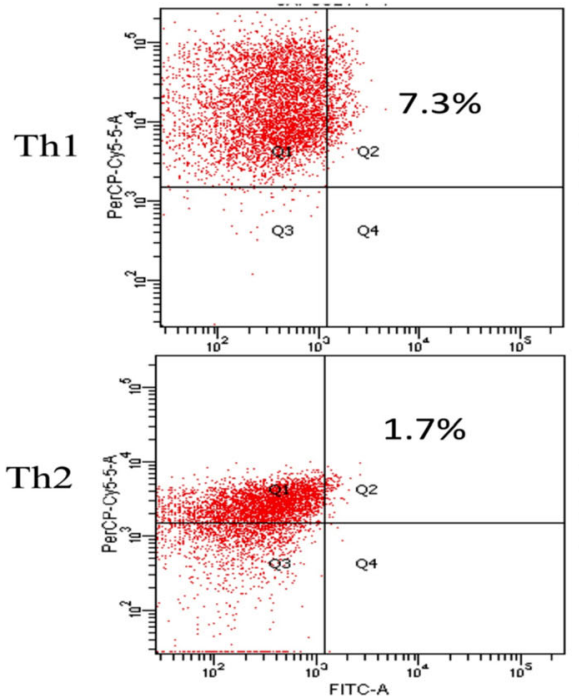

PBS

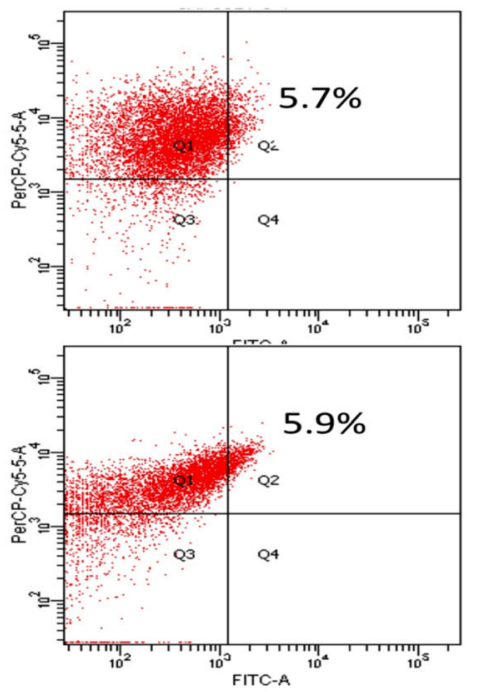

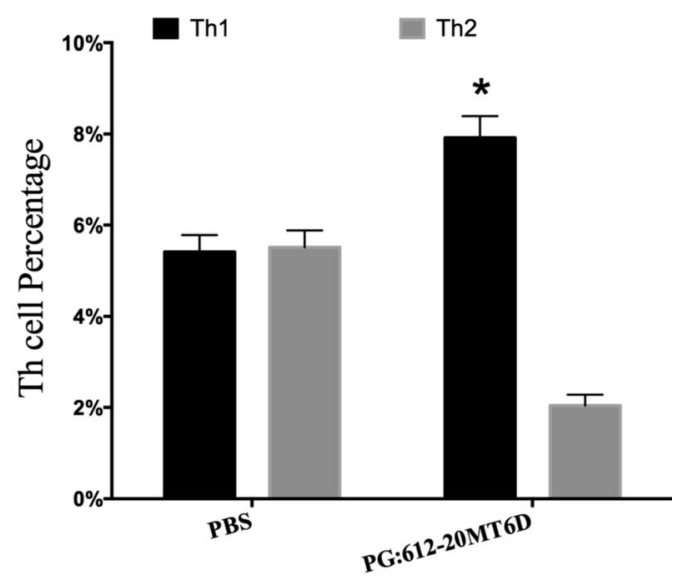

Fig. 2 FCM assay for Th1 and Th2 production in blood lymphocyte of piglets immunized with recombinant Lactobacillus casei PG:61220MT6D and $P B S$. Data are means \pm S.D. Flow cytometry analysis of

the percentage of $\mathrm{T}$ helper subtypes as gating $\mathrm{CD} 4+$ was performed. * Significant difference by student $t$ test $(P<0.05)$

\section{Application of real-time RT-PCR to clinical samples}

Piglets inoculated with virulent TGEV shed the virus for $12 \mathrm{~h}$, followed by profuse diarrhoea that led the piglets to the verge of death 1-4 days after inoculation. The TGEV load shed in the diarrhoea was $3.2 \times 10^{5}$ copies at the 12th hour, peaked at $1.72 \times 10^{6}$ copies at the 48th hour and then decreased until death in the PBS group (Fig. 5). The recombinant Lactobacillus vaccine group (PG:612-20MT6D) exhibited the same trend. The TGEV copy number was $10^{5}$ at the 12 th hour, and the copies reached a peak at the 48th hour. The copy numbers were similar and followed the same trend after reaching the peak. However, the copy numbers in the PG:612-20MT6D group were significantly lower than in the PBS group.

\section{Activation of $T$ helper cells by recombinant $L$. case $i$ after infection}

Next, we investigated the generation of Lactobacillus vaccineinduced regulatory cells after infection in piglets. As shown in
Fig. 3 Protection and antibody responses after challenge. Immunized with PG:61220MT6D and PBS piglets were orally challenged with TGEV. TGEV-challenged pigs received a 5 -mL dose of $1 \times 10^{5}$ plaqueforming unit $(\mathrm{PFU}) / \mathrm{mL}$ via oralgastric gavage on days postinoculation. Pigs (control group) were administered volumematched virus-free ST cell culture media. All piglets were euthanized at 5 days for necropsy examination

\section{Protective Efficacy}

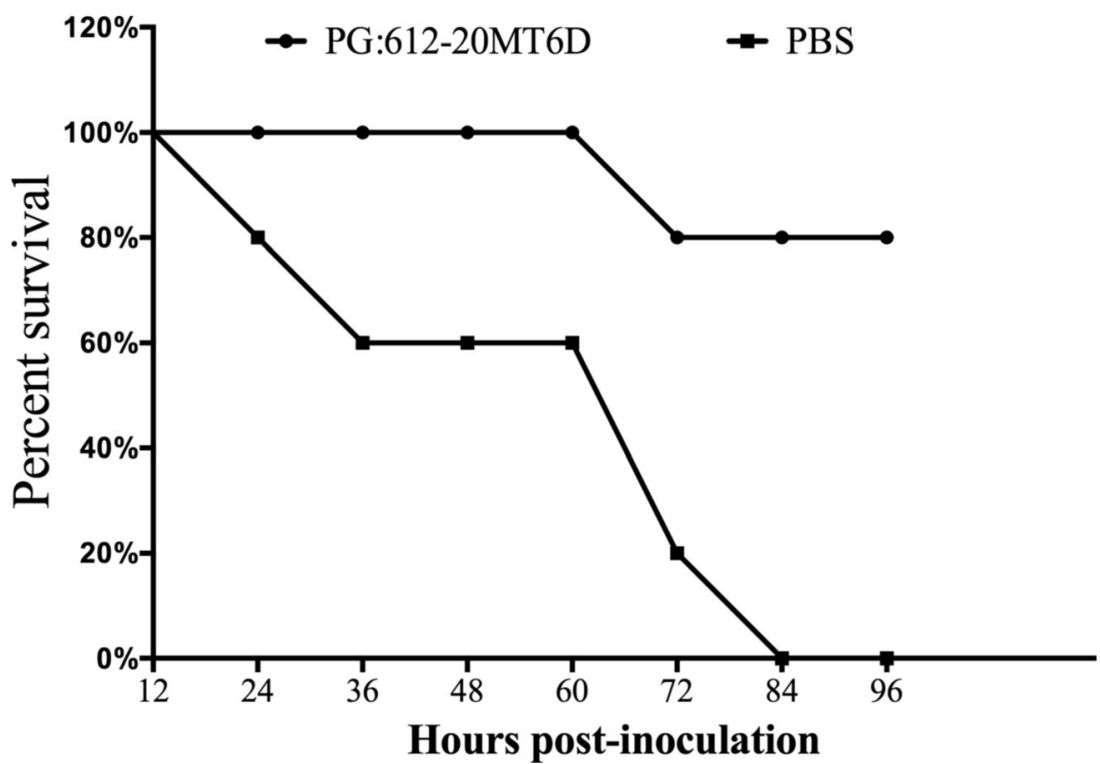


No infection group
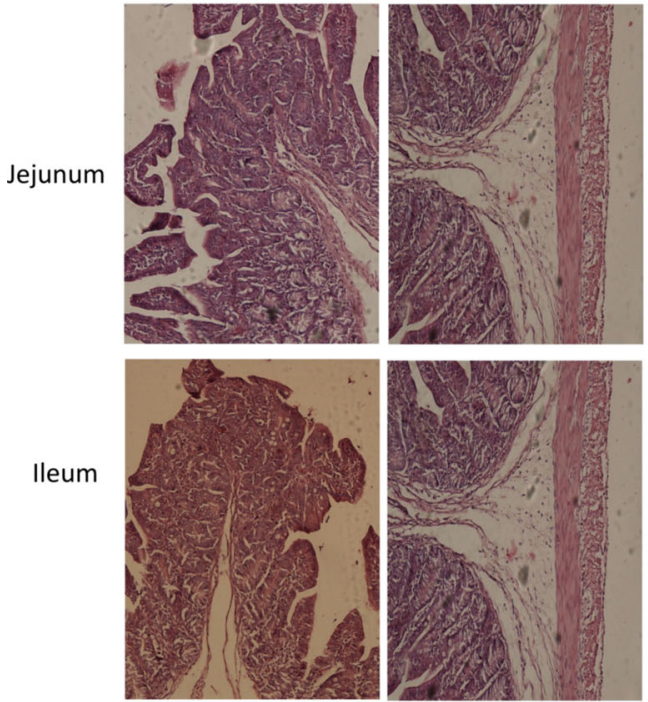

Fig. 4 Recombinant Lactobacillus casei protect piglets organs from TGEV destruction. Small intestine histology sections were stained with H\&E. Figure shows tissue of jejunum and ileum sections from piglets groups, such as recombinant Lactobacillus, no infection and infection TGEV groups. TGEV-challenged pigs received a 5 -mL dose of $1 \times 10^{5}$

Fig. 6, there was a marked increase in the production of IL-4 in CD4+ T cells. The Th1 immune response induced by the vaccine was seriously broken in favour of Th2-mediated systemic and mucosal immunity post-infection. The systemic Th2 immune response was higher than the mucosal Th2mediated immune response. After TGEV infection, the body activated more Th2 to protect itself in response to the infection.

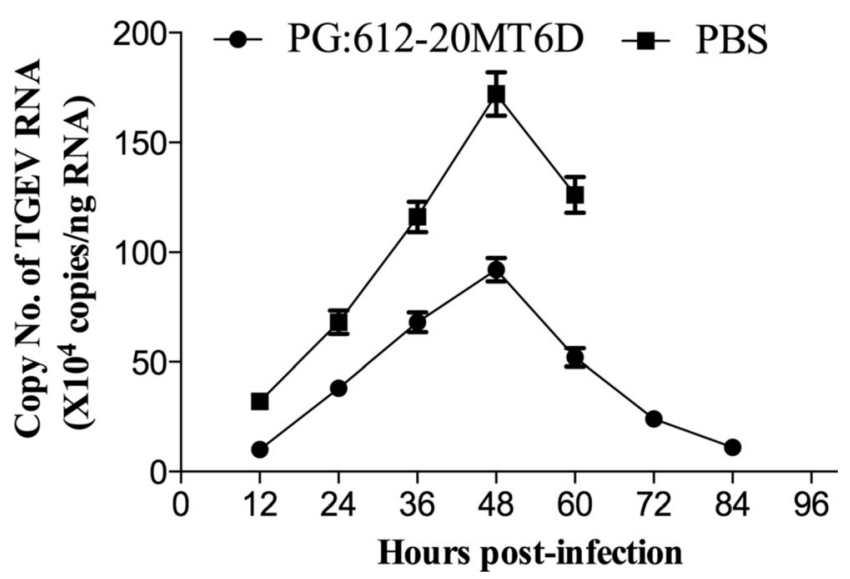

Fig. 5 Real-time RT-PCR to clinical samples. Piglets inoculated with virulent TGEV shed the virus on either $12 \mathrm{~h}$. Detection and quantification of TGEV shed in diarrhoea that persisted until death 1-4 days after inoculation, which using real-time quantification RT-PCR. Total RNA extracted from the faeces and was used for real-time qRT-PCR analysis to determine TGEV amounts. Data show the mean $\pm \mathrm{SD}$ of five piglets per group
Infection group

PG:612-20MT6D
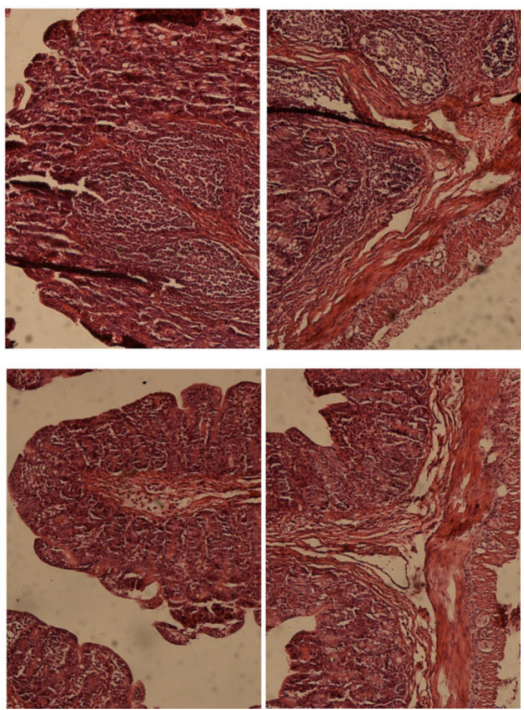

plaque-forming unit (PFU)/mL via oral-gastric gavage. Pigs (control group) were administered volume-matched virus-free cell culture media. All piglets were euthanized at $5 \mathrm{dpi}$ for necropsy examination. The histological pictures are representative of sections derived from five piglets per group. Original magnifications: $\times 100$

\section{Cytokines and TLR expression}

As shown in Fig. 7, Toll-like receptor (TLR) expression was detected in the splenic lymphocytes (SL) and mesenteric lymph node cells (ML) in the piglets in the PBS and vaccine groups. TLR-2 was higher in the vaccine group than in the PBS group. In contrast, there were no significant differences between TLR-4 and TLR-9. However, the three TLRs exhibited the same trends in the mesenteric lymph node cells compared with the PBS and Lactobacillus vaccine. TGEV infection induced TLR expression and especially enhanced TLR-2 and TLR-4 expression, but the expression levels in the Lactobacillus vaccine group were significantly lower than the levels in the PBS group. Cytokine expression in the splenic lymphocytes and mesenteric lymph node cells was also analysed and compared in our study. The IFN- $\gamma$, IL-4 and IL-17 levels in the splenic lymphocytes from the Lactobacillus vaccine group showed marked changes, whereas no significant difference was observed in the level of TGF- $\beta$. TGEV infection stimulated cytokine expression in the PBS group, including IFN- $\gamma$ and IL-4. The vaccine group did not express a notably higher level of IFN- $\gamma$ and IL-4 compared with the PBS group. The TGF- $\beta$ expression level in the mesenteric lymph node cells was lower in the vaccine group than in the PBS group; the same trend was observed with IFN- $\gamma$ and IL-4. The vaccine group provoked higher IL17 expression than the PBS group following TGEV infection. The IL-17 expression levels in both the splenic lymphocytes 
$P G: 612-20 M T 6 D$
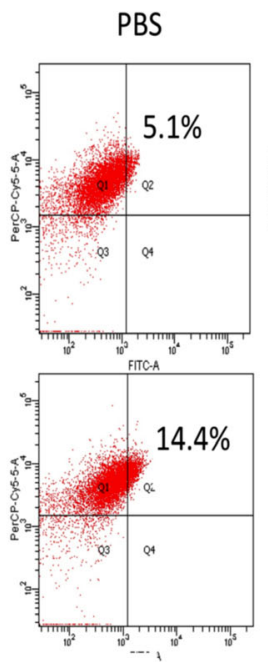

Splenic lymphocyte
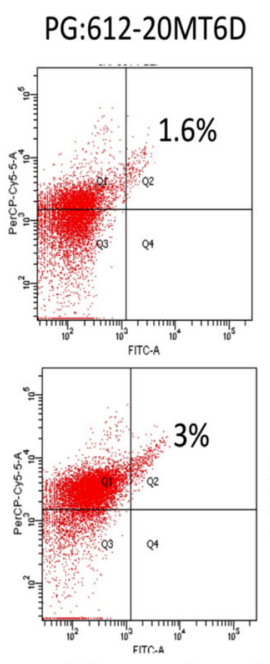

Mesenteric node lymphocyte
PBS
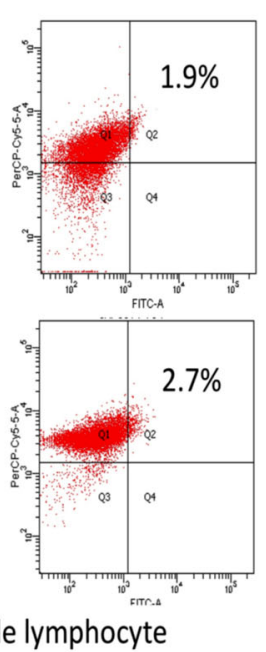

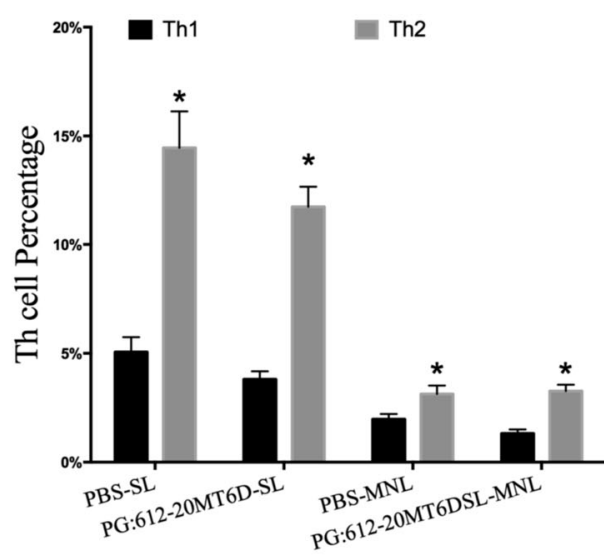

lymphocyte (ML). Data are means \pm S.D. Flow cytometry analysis of the percentage of $\mathrm{T}$ helper subtypes as gating CD4+ was performed. * Significant difference by student $t$ test $(P<0.05)$

result, we find that the two-dose immunization is still the best immunization plan. The reason for the diarrhoea after immunization is the overdose of Lactobacillus, which is a type of microbe that causes a disturbance in the intestinal microbial flora for short time. Many enteric pathogens must first adhere to the intestinal epithelial cells to initiate intestinal disease. Limiting access of the pathogens to intestinal epithelial cells is one strategy to prevent disease that has been investigated previously. For example, the competitive inhibition of bacterial adherence by mimicry of receptors on the apical surface of enterocytes using oral administration of sialylated glycoproteins has been shown to protect newborn calves from the enterotoxigenic E. coli strain K99 (Mouricout et al. 1990). Lactobacillus must colonize the gut soon after birth; therefore, the vaccine could play a role in non-specific immunity. Probiotic strains with a high adherence capacity have been demonstrated to enhance the immunoglobulin A response to rotavirus (Kaila et al. 1992).

In this study, we observed a significant increase in the anti-TGEV IgA titre in the intestinal tract of piglets administered recombinant $L$. casei. Furthermore, we showed that the diarrhoea state of piglets administered $L$. casei was significantly lower than that of piglets administered saline. In the murine model of IFV infection, the virus moves from the upper respiratory tract to the lower respiratory tract (Hori et al. 2001). HRV-vaccinated and Lactobacillus acidophilus-fed pigs had a significantly higher magnitude of HRV-specific IgA and IgG antibody-secreting cell responses in the ileum and serum IgG antibody and virus neutralizing antibody titres compared to HRV-vaccinated pigs without $L$. acidophilus colonization (Zhang et al. 2008). Our immunization stimulated the same systemic 


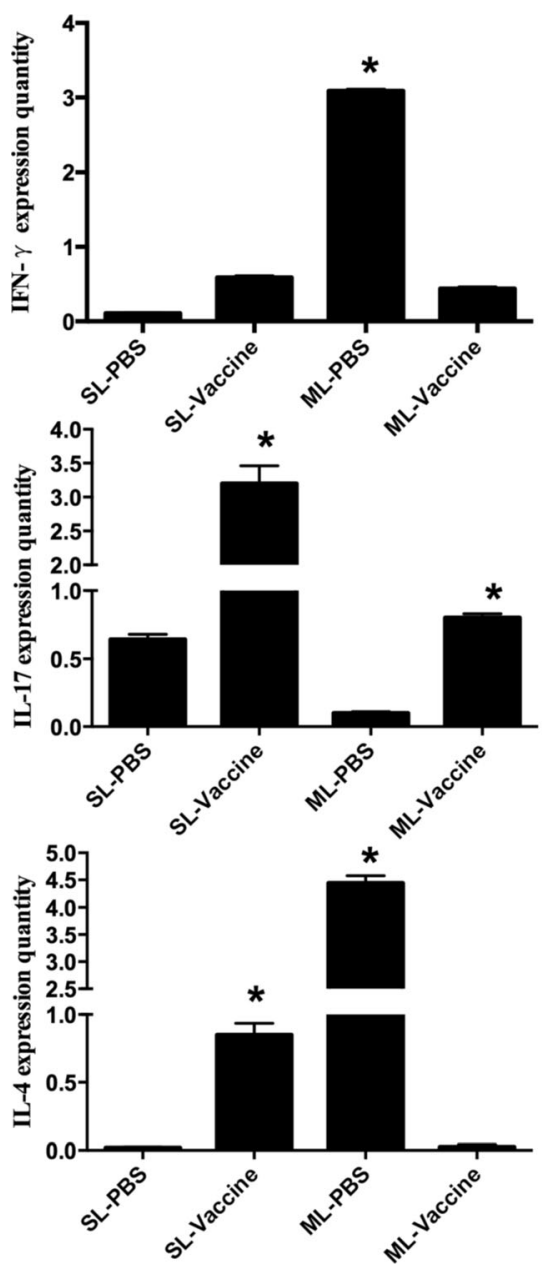

Fig. 7 Cytokines and TLR expression. The RNA of splenic lymphocyte (SL) and mesenteric lymphocyte (ML) in immunized piglets, PG:61220MT6D and PBS groups, were used to analysed the cytokines and TLR expression. IFN- $\gamma$, IL-4, IL-17, TGF- $\beta$, TLRs expressing were detected in splenic lymphocyte (SL) and mesenteric lymph cells (ML) piglets,
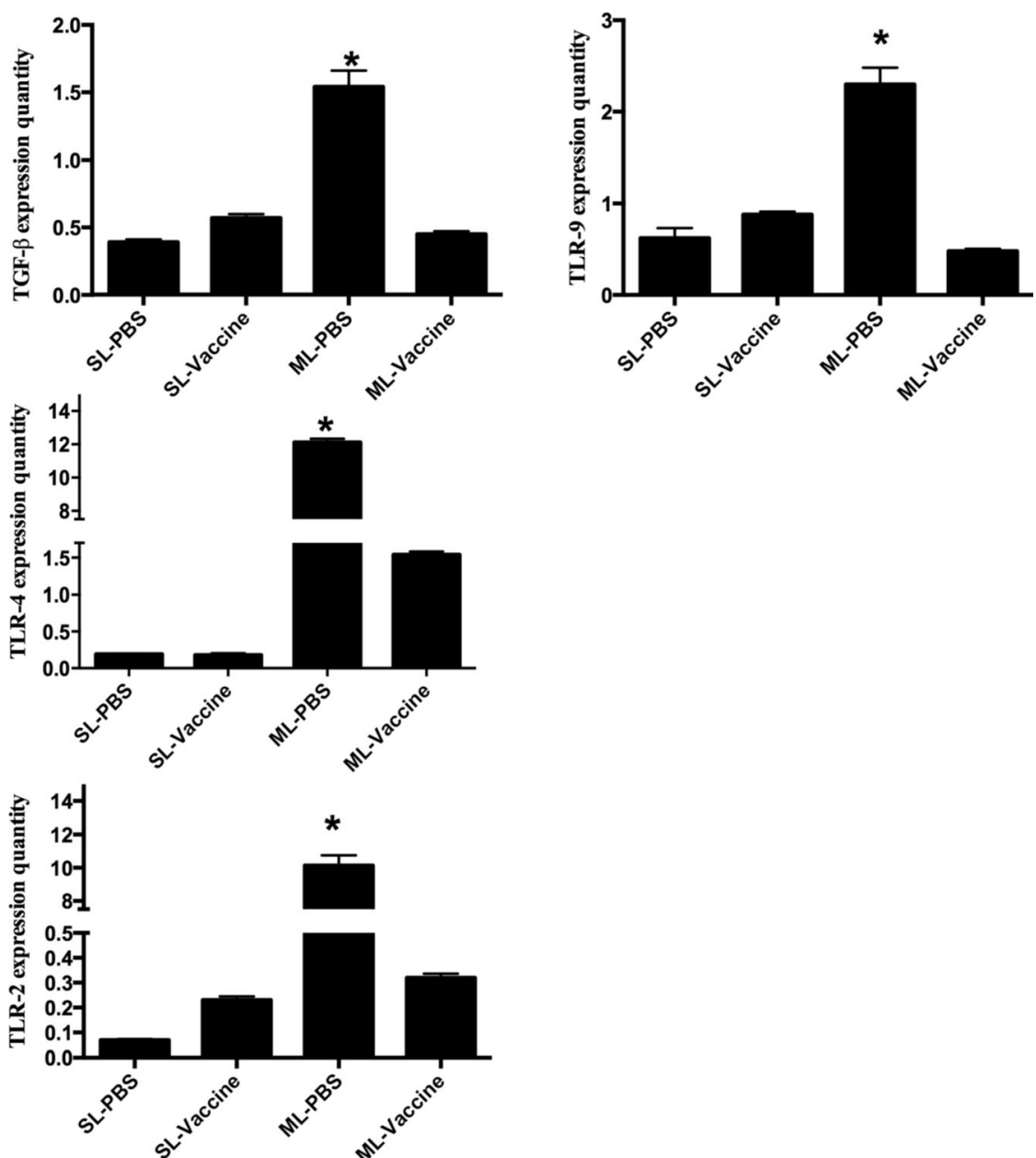

such as PBS and vaccine groups. *Significant difference by student $t$ test $(P<0.05)$. Data shown were compared using one-way analysis of variance (ANOVA) followed by Duncan's multiple range test. Representative for three independent experiments

Lactobacillus induced a mucosal immune responses in the piglets. Taken together, our data show the tremendous potential for recombinant Lactobacillus to enable rapid and effective treatment for TGEV infection with intestinal tropism in piglets.

We evaluated the specific T cell immune responses induced by the recombinant $L$. casei vaccine compared with the PBS control group in the piglets. We demonstrated that $L$. casei significantly enhanced the immunogenicity of the TGEV vaccine as indicated by the significantly higher magnitude of specific IFN- $\gamma$-producing CD4+ T cell responses. There has reported that mice fed recombinant $L$. casei with the adjuvant MDP and tuftsin have significantly higher Th1 and Th17 production than control group mice (Jiang et al. 2014). These results were the same and indicated that $L$. casei had a strong potentiating effect on both the cellular and humoral immunity induced by the oral $L$. casei vaccine. Similarly, a previous study showed that oral intake of $L$. fermentum CECT5716 recombinant Lactobacillus, indicating that recombinant 
significantly enhanced serum Th1 type cytokine production and influenza-specific IgA antibody responses to an intramuscular influenza vaccine in adults (Olivares et al. 2007).

The mesenteric lymph nodes were primarily used to analyse TGEV infection and the immunoprotection provided by the Lactobacillus vaccine in terms of mucosal immunization and infection. IFN- $\gamma$ induction by TGEV results from interactions between an outer membrane domain of El and the PBMC membrane (Charley and Laude 1988); however, these authors did not study the expression of IL-4 by PBMCs. The expression of IFN- $\gamma$ was higher than IL-4 in the immunized group, and the Th1/Th2 balance was broken in our study. After immunization with recombinant Lactobacillus, IFN- $\gamma$ played a major role in the mucosal immune response. However, after TGEV infection, the systemic and local immune responses shifted from Th1 to Th2. The systemic humoral immune response was stronger than the cellular immune response after TGEV infection. This is the first study to demonstrate that TGEV infection polarized the immune response to Th2 immunity and that recombinant Lactobacillus could weaken TGEV infection in the form of Th2 immunity. From these results, we found that the immunization did not polarize Th2 immunity more seriously compared to the PBS control group. The proteinbased SARS coronavirus vaccine boost induced similar levels of Th1 and neutralizing antibody responses that protected vaccinated mice from subsequent SARS-CoV challenges but induced stronger Th2 and CTL responses (Zheng et al. 2008). The UV-inactivated SARS coronavirus vaccine retained its immunogenicity and promoted Th2type immune responses (Tsunetsugu-Yokota et al. 2007). The activation of Th2 responses such as IL-10 stimulate B cell proliferation, which can produce specific and nonspecific anti-infection antibodies (Grodeland et al. 2015); similarly, both $\mathrm{T}$ and $\mathrm{B}$ cells have functions following Lactobacillus vaccination. The production of IL-9 by Th2 cells results in the proliferation of mast cell growth, and IL13 stimulates epithelial cell growth (Tukler Henriksson et al. 2015). The proliferation of epithelial cells is crucial for TGEV infection. The Th2 response could also stimulate the production of mucus by epithelial cells (Zhang et al. 2015).

IL-17 cells play an essential role in MHV-induced immunopathology, and IFN- $\gamma$ is important for maintaining the immune balance between Th1 and Th17 responses during acute viral infection (Yang et al. 2011). However, the Th1/Th2 balance in the negative control group was different than the balance in the immunized group. TGEV affects both systemic and local cellular immunity without immunization. IL17 has been reported to have both pro- and anti-inflammatory effects (Lafdil et al. 2009; Nagata et al. 2008). Our results showed that the immunized piglets provoked IL-17 expression form both the systematic and mucosal immune responses after TGEV infection. Compared with the mucosal immune response, IL-17 expression in the mesenteric lymph nodes was markedly lower than the expression in the spleen cells. However, IL-17 expression in the PBS group was lower than the expression level in the immunized groups, indicating that the Lactobacillus vaccine group activated IL-17 expression during TGEV infection. Swine-origin influenza A virus-infected patients exhibited rapid lymphopenia, $\mathrm{T}$ cell activation and a preferential loss of the Th17 subset during the early stage of acute infection (Jiang et al. 2010), which was consistent with the first reports that swine-origin virus inhibited Th17 proliferation after infection. Our study also found the same phenomenon after coronavirus infection in swine compared with the immunized group. The most important finding was that the oral recombinant Lactobacillus vaccine stimulated Th17 cell proliferation. The proliferation was involved in cytokine and chemokine production, neutrophil recruitment, promotion of $\mathrm{T}$ cell priming and antibody production (Dirisala et al. 2013). Th17 responses are protective against lethal influenza virus infection in IL-10deficient mice (McKinstry et al. 2009). In contrast, a deleterious role of IL-17 has been proposed to contribute to the acute lung injury associated with IL-17-mediated neutrophil recruitment during influenza virus infection (Crowe et al. 2009). There were significant changes in the IL-4 and IFN- $\gamma$ expression levels in the mesenteric lymph node cells compared with the PBS group. Moreover, IL-4 expression was higher than IFN- $\gamma$ expression in the spleen cells. The expression of IL- 4 and IFN- $\gamma$ indicated that the recombinant Lactobacillus effectively inhibited inflammation after TGEV infection.

In this study, we also evaluated TLR-2, TLR-4 and TLR-9 expression in piglets immunized with recombinant L. case $i$ and then challenged with TGEV. Previously, TLR expression in pigs has been studied only at the mRNA level in lymphocytes using real-time PCR because antibodies against porcine TLRs are not currently available. TGEV infection did not induce TLR-2, TLR-4 and TLR9 expression in the spleen cells. However, TLR expression was significantly different in the mesenteric lymph node cells compared with the PBS and Lactobacillus vaccine groups. TLR expression was extremely high in the PBS group compared with the other groups. The cytokine and TLR expression levels in the splenic lymphocytes are indicators of systemic immunity, whereas the expression in the mesenteric lymph node cells was associated with the local and mucosal immune responses. The expression levels of all TLRs in mesenteric lymph node cells were higher in the PBS group than the vaccine group, suggesting that the Lactobacillus vaccine effectively inhibited TLR expression. The recombinant Lactobacillus groups 
exhibited jejuna and ilea with lymphocyte proliferation in the lamina propria. Transmissible gastroenteritis (TGE) coronavirus infection resulted in antibody production from primed mesenteric lymph node cells following an in vitro boost with viral antigen (Berthon et al. 1990); as an intestinal infectious disease, TGEV would attack the intestinal tissue and local immune system. Exposure of pigs to TGEV or PRCV results in distinct disease patterns related to differences in tissue tropism (Saif 1996). However, the increased frequencies of TLR-2 and TLR-9 expression in pigs may simply be due to the significantly higher counts of $L$. casei or MDP and tuftsin, which may translate to an increased magnitude of TLR agonists available to stimulate the host mucosal immune system. It is likely that the higher LAB count in the LAB plus HRV group played a more pertinent role in the significant increases in TLR2 and TLR9 expression (Wen et al. 2009). Taken together, TGEV immune protection was primarily dependent on the mucosal immune response. Systemic immunity did not play a key role after TGEV infection. Interestingly, IL-17 expression in the vaccine group was significant higher than IL-17 expression in the PBS group challenged with TGEV, and Th17 played an anti-inflammatory role in mucosal immunity. IL-17 also stimulated intestinal epithelial cell differentiation and growth.

In conclusion, our study suggests that the recombinant Lactobacillus vaccine provokes specific mucosal and systemic immune responses to protect piglets from infection. IgA played a dominant role in the mucosal immune response after TGEV challenge. Therefore, the histopathology and RNA copy numbers directly demonstrated that the Lactobacillus vaccine was effective for TGEV infection. The protective efficacy was significantly higher, which would have great value in practice. Moreover, although the recombinant Lactobacillus vaccine-induced Th1 immunity, the immune balance was broken by TGEV infection; thus, the immunized piglets initiated a Th2 immune response against TGEV infection. Taken together, we showed that IL-17 signalling was vital for Lactobacillus vaccine immunized piglets infected with TGEV. The Lactobacillus vaccine also inhibited TLR expression, which indicated that most of virus was neutralized because the TLRs were not activated by the virus.

Acknowledgments This work was supported by the National Natural Science Foundation of China (31472226).

\section{Compliance with ethical standards}

Conflict of interest The authors declare that they have no competing interests.

Ethical statement The piglets were handled and maintained under strict ethical conditions according to international recommendations for animal welfare. This article does not contain any studies with human participants performed by any of the authors.

\section{References}

Anton IM, Sune C, Meloen RH, Borras-Cuesta F, Enjuanes L (1995) A transmissible gastroenteritis coronavirus nucleoprotein epitope elicits $\mathrm{T}$ helper cells that collaborate in the in vitro antibody synthesis to the three major structural viral proteins. Virology 212(2):746751. doi:10.1006/viro.1995.1535

Anton IM, Gonzalez S, Bullido MJ, Corsin M, Risco C, Langeveld JP, Enjuanes L (1996) Cooperation between transmissible gastroenteritis coronavirus (TGEV) structural proteins in the in vitro induction of virus-specific antibodies. Virus Res 46(1-2):111-124

Bengmark S, Gil A (2006) Bioecological and nutritional control of disease: prebiotics, probiotics and synbiotics. Nutr Hosp 21(Suppl 2): 72-84, 73-86

Berthon P, Bernard S, Salmon H, Binns RM (1990) Kinetics of the in vitro antibody response to transmissible gastroenteritis (TGE) virus from pig mesenteric lymph node cells, using the ELISASPOT and ELISA tests. J Immunol Methods 131(2):173-182

Blum S, Schiffrin EJ (2003) Intestinal microflora and homeostasis of the mucosal immune response: implications for probiotic bacteria? Curr Issues Intest Microbiol 4(2):53-60

Braat H, Rottiers P, Hommes DW, Huyghebaert N, Remaut E, Remon JP, van Deventer SJ, Neirynck S, Peppelenbosch MP, Steidler L (2006) A phase I trial with transgenic bacteria expressing interleukin-10 in Crohn's disease. Clin Gastroenterol Hepatol 4(6):754-759. doi:10. 1016/j.cgh.2006.03.028

Cavanagh D (1997) Nidovirales: a new order comprising Coronaviridae and Arteriviridae. Arch Virol 142(3):629-633

Charley B, Laude H (1988) Induction of alpha interferon by transmissible gastroenteritis coronavirus: role of transmembrane glycoprotein E1. J Virol 62(1):8-11

Chen CC, Baylor M, Bass DM (1993) Murine intestinal mucins inhibit rotavirus infection. Gastroenterology 105(1):84-92

Corthesy B, Gaskins HR, Mercenier A (2007) Cross-talk between probiotic bacteria and the host immune system. J Nutr 137(3 Suppl 2): 781S-790S

Crowe CR, Chen K, Pociask DA, Alcorn JF, Krivich C, Enelow RI, Ross TM, Witztum JL, Kolls JK (2009) Critical role of IL-17RA in immunopathology of influenza infection. J Immunol 183(8):53015310. doi:10.4049/jimmunol.0900995

de Vrese M, Marteau PR (2007) Probiotics and prebiotics: effects on diarrhea. J Nutr 137(3 Suppl 2):803S-811S

Di-Qiu L, Xin-Yuan Q, Jun-Wei G, Li-Jie T, Yan-Ping J, Yi-Jing L (2011) Construction and characterization of Lactobacillus pentosus expressing the D antigenic site of the spike protein of Transmissible gastroenteritis virus. Can J Microbiol 57(5):392-397. doi:10.1139/ W11-027

Dirisala VR, Jeevan A, Ramasamy SK, McMurray DN (2013) Molecular cloning, expression, and in silico structural analysis of guinea pig IL-17. Mol Biotechnol 55(3):277-287. doi:10.1007/s12033-0139679-Z

Dock DB, Aguilar-Nascimento JE, Latorraca MQ (2004) Probiotics enhance the recovery of gut atrophy in experimental malnutrition. Biocell 28(2):143-150

Friedrich MJ (2013) Genomes of microbes inhabiting the body offer clues to human health and disease. JAMA 309(14):1447-1449. doi:10. 1001/jama.2013.2824 
Grodeland G, Fossum E, Bogen B (2015) Polarizing T and B cell responses by APC-targeted subunit vaccines. Front Immunol 6:367. doi:10.3389/fimmu.2015.00367

Gunzer F, Hennig-Pauka I, Waldmann KH, Sandhoff R, Grone HJ, Kreipe HH, Matussek A, Mengel M (2002) Gnotobiotic piglets develop thrombotic microangiopathy after oral infection with enterohemorrhagic Escherichia coli. Am J Clin Pathol 118(3): 364-375. doi:10.1309/UMW9-D06Q-M94Q-JGH2

Hori T, Kiyoshima J, Shida K, Yasui H (2001) Effect of intranasal administration of Lactobacillus casei Shirota on influenza virus infection of upper respiratory tract in mice. Clin Diagn Lab Immunol 8(3): 593-597. doi:10.1128/CDLI.8.3.593-597.2001

Isolauri E, Kirjavainen PV, Salminen S (2002) Probiotics: a role in the treatment of intestinal infection and inflammation? Gut 50(Suppl 3): III54-III59

Jiang TJ, Zhang JY, Li WG, Xie YX, Zhang XW, Wang Y, Jin L, Wang FS, Zhao M (2010) Preferential loss of Th17 cells is associated with CD4 T cell activation in patients with 2009 pandemic H1N1 swineorigin influenza A infection. Clin Immunol 137(3):303-310. doi:10. 1016/j.clim.2010.07.010

Jiang X, Yu M, Qiao X, Liu M, Tang L, Jiang Y, Cui W, Li Y (2014) Upregulation of MDP and tuftsin gene expression in Th1 and Th17 cells as an adjuvant for an oral Lactobacillus casei vaccine against anti-transmissible gastroenteritis virus. Appl Microbiol Biotechnol 98(19):8301-8312. doi:10.1007/s00253-014-5893-2

Kaila M, Isolauri E, Soppi E, Virtanen E, Laine S, Arvilommi H (1992) Enhancement of the circulating antibody secreting cell response in human diarrhea by a human Lactobacillus strain. Pediatr Res 32(2): 141-144. doi:10.1203/00006450-199208000-00002

Kararli TT (1995) Comparison of the gastrointestinal anatomy, physiology, and biochemistry of humans and commonly used laboratory animals. Biopharm Drug Dispos 16(5):351-380

Kiros TG, van Kessel J, Babiuk LA, Gerdts V (2011) Induction, regulation and physiological role of IL-17 secreting helper T-cells isolated from PBMC, thymus, and lung lymphocytes of young pigs. Vet Immunol Immunopathol 144(3-4):448-454. doi:10.1016/j. vetimm.2011.08.021

Lafdil F, Wang H, Park O, Zhang W, Moritoki Y, Yin S, Fu XY, Gershwin ME, Lian ZX, Gao B (2009) Myeloid STAT3 inhibits T cellmediated hepatitis by regulating $\mathrm{T}$ helper 1 cytokine and interleukin-17 production. Gastroenterology 137(6):2125-2135. doi:10.1053/j.gastro.2009.08.004, e1-2

Lavelle EC, O'Hagan DT (2006) Delivery systems and adjuvants for oral vaccines. Expert Opin Drug Deliv 3(6):747-762. doi:10.1517/ 17425247.3.6.747

Lee BM, Han YW, Kim SB, Rahman MM, Uyangaa E, Kim JH, Roh YS, Kim B, Han SB, Hong JT, Kim K, Eo SK (2011) Enhanced protection against infection with transmissible gastroenteritis virus in piglets by oral co-administration of live attenuated Salmonella enterica serovar Typhimurium expressing swine interferon-alpha and interleukin-18. Comp Immunol Microbiol Infect Dis 34(4):369-380. doi:10.1016/j.cimid.2011.05.001

Liu D, Wang X, Ge J, Liu S, Li Y (2011) Comparison of the immune responses induced by oral immunization of mice with Lactobacillus casei-expressing porcine parvovirus VP2 and VP2 fused to Escherichia coli heat-labile enterotoxin B subunit protein. Comp Immunol Microbiol Infect Dis 34(1):73-81. doi:10.1016/j.cimid. 2010.02.004

Malik DK, Baboota S, Ahuja A, Hasan S, Ali J (2007) Recent advances in protein and peptide drug delivery systems. Curr Drug Deliv 4(2): 141-151

McKinstry KK, Strutt TM, Buck A, Curtis JD, Dibble JP, Huston G, Tighe M, Hamada H, Sell S, Dutton RW, Swain SL (2009) IL-10 deficiency unleashes an influenza-specific Th17 response and enhances survival against high-dose challenge. J Immunol 182(12): 7353-7363. doi:10.4049/jimmunol.0900657
Moore-Connors JM, Fraser R, Halperin SA, Wang J (2013) CD4(+ )CD25(+)Foxp3(+) regulatory $\mathrm{T}$ cells promote Th17 responses and genital tract inflammation upon intracellular Chlamydia muridarum infection. J Immunol 191(6):3430-3439. doi:10.4049/jimmunol. 1301136

Mouricout M, Petit JM, Carias JR, Julien R (1990) Glycoprotein glycans that inhibit adhesion of Escherichia coli mediated by K99 fimbriae: treatment of experimental colibacillosis. Infect Immun 58(1):98 106

Nagata T, McKinley L, Peschon JJ, Alcorn JF, Aujla SJ, Kolls JK (2008) Requirement of IL-17RA in Con A induced hepatitis and negative regulation of IL-17 production in mouse T cells. J Immunol 181(11): 7473-7479

Olivares M, Diaz-Ropero MP, Sierra S, Lara-Villoslada F, Fonolla J, Navas M, Rodriguez JM, Xaus J (2007) Oral intake of Lactobacillus fermentum CECT5716 enhances the effects of influenza vaccination. Nutrition 23(3):254-260. doi:10.1016/j.nut.2007. 01.004

Oswald IP, Desautels C, Laffitte J, Fournout S, Peres SY, Odin M, Le Bars P, Le Bars J, Fairbrother JM (2003) Mycotoxin fumonisin B1 increases intestinal colonization by pathogenic Escherichia coli in pigs. Appl Environ Microbiol 69(10):5870-5874

Ouwehand AC (2007) Antiallergic effects of probiotics. J Nutr 137(3 Suppl 2):794S-797S

Qiao X, Li G, Wang X, Li X, Liu M, Li Y (2009) Recombinant porcine rotavirus VP4 and VP4-LTB expressed in Lactobacillus casei induced mucosal and systemic antibody responses in mice. BMC Microbiol 9:249. doi:10.1186/1471-2180-9-249

Ruan X, Zhang W (2013) Oral immunization of a live attenuated Escherichia coli strain expressing a holotoxin-structured adhesintoxoid fusion (1FaeG-FedF-LTA(2):5LTB) protected young pigs against enterotoxigenic E. coli (ETEC) infection. Vaccine 31(11): 1458-1463. doi:10.1016/j.vaccine.2013.01.030

Saarela M, Mogensen G, Fonden R, Matto J, Mattila-Sandholm T (2000) Probiotic bacteria: safety, functional and technological properties. J Biotechnol 84(3):197-215

Saif LJ (1996) Mucosal immunity: an overview and studies of enteric and respiratory coronavirus infections in a swine model of enteric disease. Vet Immunol Immunopathol 54(1-4):163-169

Sartor RB (2005) Probiotic therapy of intestinal inflammation and infections. Curr Opin Gastroenterol 21(1):44-50

Schwegmann-Wessels C, Herrler G (2006) Transmissible gastroenteritis virus infection: a vanishing specter. Dtsch Tierarztl Wochenschr 113(4):157-159

Sheil B, Shanahan F, O'Mahony L (2007) Probiotic effects on inflammatory bowel disease. J Nutr 137(3 Suppl 2):819S-824S

Siddell SG, Anderson R, Cavanagh D, Fujiwara K, Klenk HD, Macnaughton MR, Pensaert M, Stohlman SA, Sturman L, van der Zeijst BA (1983) Coronaviridae. Intervirology 20(4):181-189

Superti F, Marziano ML, Tinari A, Donelli G (1993) Effect of polyions on the infectivity of SA-11 rotavirus in LCC-MK2 cells. Comp Immunol Microbiol Infect Dis 16(1):55-62

Tadros T, Traber DL, Heggers JP, Herndon DN (2003) Effects of interleukin-1alpha administration on intestinal ischemia and reperfusion injury, mucosal permeability, and bacterial translocation in burn and sepsis. Ann Surg 237(1):101-109. doi:10.1097/01.SLA. 0000041039.16815 .69

Tsunetsugu-Yokota Y, Ato M, Takahashi Y, Hashimoto S, Kaji T, Kuraoka M, Yamamoto K, Mitsuki YY, Yamamoto T, Oshima M, Ohnishi K, Takemori T (2007) Formalin-treated UV-inactivated SARS coronavirus vaccine retains its immunogenicity and promotes Th2-type immune responses. Jpn J Infect Dis 60(2-3):106-112

Tukler Henriksson J, Coursey TG, Corry DB, De Paiva CS, Pflugfelder SC (2015) IL-13 stimulates proliferation and expression of mucin and immunomodulatory genes in cultured conjunctival goblet cells. 
Invest Ophthalmol Vis Sci 56(8):4186-4197. doi:10.1167/iovs.1415496

Villena J, Racedo S, Aguero G, Alvarez S (2006) Yoghurt accelerates the recovery of defence mechanisms against Streptococcus pneumoniae in protein-malnourished mice. Br J Nutr 95(3):591-602

Wells JM, Mercenier A (2008) Mucosal delivery of therapeutic and prophylactic molecules using lactic acid bacteria. Nat Rev Microbiol 6(5):349-362. doi:10.1038/nrmicro1840

Wen K, Azevedo MS, Gonzalez A, Zhang W, Saif LJ, Li G, Yousef A, Yuan L (2009) Toll-like receptor and innate cytokine responses induced by lactobacilli colonization and human rotavirus infection in gnotobiotic pigs. Vet Immunol Immunopathol 127(3-4):304-315. doi:10.1016/j.vetimm.2008.10.322

Yang W, Ding X, Deng J, Lu Y, Matsuda Z, Thiel A, Chen J, Deng $H$, Qin Z (2011) Interferon-gamma negatively regulates Th17mediated immunopathology during mouse hepatitis virus infection. J Mol Med (Berl) 89(4):399-409. doi:10.1007/s00109_ 010-0711-5

Yigang XU, Yijing LI (2008) Construction of recombinant Lactobacillus casei efficiently surface displayed and secreted porcine parvovirus VP2 protein and comparison of the immune responses induced by oral immunization. Immunology 124(1):68-75. doi:10.1111/j.13652567.2007.02738.x

Yuan TL, Zhu YH, Shi M, Li TT, Li N, Wu GY, Bazer FW, Zang JJ, Wang FL, Wang JJ (2015) Within-litter variation in birth weight: impact of nutritional status in the sow. J Zhejiang Univ Sci B 16(6):417-435. doi:10.1631/jzus.B1500010

Zhang W, Azevedo MS, Wen K, Gonzalez A, Saif LJ, Li G, Yousef AE, Yuan L (2008) Probiotic Lactobacillus acidophilus enhances the immunogenicity of an oral rotavirus vaccine in gnotobiotic pigs. Vaccine 26(29-30):3655-3661. doi:10.1016/j.vaccine.2008.04.070

Zhang Y, Wang X, Wang H, Jiao J, Li Y, Fan E, Zhang L, Bachert C (2015) TMEM16A-mediated mucin secretion in il-13-induced nasal epithelial cells from chronic rhinosinusitis patients. Allergy Asthma Immunol Res 7(4):367-375. doi:10.4168/aair.2015.7.4.367

Zheng BJ, Du LY, Zhao GY, Lin YP, Sui HY, Chan C, Ma S, Guan Y, Yuen KY (2008) Studies of SARS virus vaccines. Hong Kong Med J 14(Suppl 4):39-43

Zhou X, Chen Q, Moore J, Kolls JK, Halperin S, Wang J (2009) Critical role of the interleukin-17/interleukin-17 receptor axis in regulating host susceptibility to respiratory infection with Chlamydia species. Infect Immun 77(11):5059-5070. doi:10.1128/IAI.00403-09 